\title{
BMJ Open Instruments for measuring nursing research competence: a protocol for a scoping review
}

\author{
Qirong Chen (D) , ${ }^{1}$ Chongmei Huang (i) , ${ }^{1}$ Aimee R Castro (id , ${ }^{2}$ Siyuan Tang (i) ${ }^{1}$
}

To cite: Chen $Q$, Huang $C$, Castro AR, et al. Instruments for measuring nursing research competence: a protocol for a scoping review. BMJ Open 2021;11:e042325. doi:10.1136/ bmjopen-2020-042325

- Prepublication history and supplemental material for this paper are available online. To view these files, please visit the journal online (http://dx.doi org/10.1136/bmjopen-2020042325).

Received 01 July 2020 Revised 23 November 2020 Accepted 27 January 2021
Check for updates

(C) Author(s) (or their employer(s)) 2021. Re-use permitted under CC BY-NC. No commercial re-use. See rights and permissions. Published by BMJ.

${ }^{1}$ Xiangya School of Nursing, Central South University, Changsha, Hunan, China 'Ingram School of Nursing, McGill University, Montreal, Québec, Canada

Correspondence to Professor Siyuan Tang; tsycongcong@126.com and Dr Chongmei Huang; 15874162760@163.com

\section{ABSTRACT}

Introduction Nursing research competence of nursing personnel has received much attention in recent years, as nursing has developed as both an independent academic discipline and an evidence-based practiing profession. Instruments for appraising nursing research competence are important, as they can be used to assess nursing research competence of the target population, showing changes of this variable over time and measuring the effectiveness of interventions for improving nursing research competence. There is a need to map the current state of the science of the instruments for nursing research competence, and to identify well validated and reliable instruments. This paper describes a protocol for a scoping review to identify, evaluate, compare and summarise the instruments designed to measure nursing research competence.

Methods and analysis The scoping review will be conducted following Arksey and 0'Malley's methodological framework and Levac et als additional recommendations for applying this framework. The scoping review will be reported according to the Preferred Reporting Items for Systematic Reviews and Meta-Analyses extension for Scoping Reviews checklist. The protocol is registered through the Open Science Framework (https://osf. io/ksh43/). Eight English databases and two Chinese databases will be searched between 1 December 2020 and 31 December 2020 to retrieve manuscripts which include instrument(s) of nursing research competence. The literature screening and data extraction will be conducted by two researchers, independently. A third researcher will be involved when consensus is needed. The COnsensusbased Standards for the selection of health Measurement INstruments methodology will be used to evaluate the methodological quality of the included studies on measurement properties of the instruments, as well as the quality of all the instruments identified.

Ethics and dissemination Ethical approval is not needed. We will disseminate the findings through a conference focusing on nursing research competence and publication of the results in a peer-reviewed journal.

\section{INTRODUCTION}

Nursing research is important for the development of nursing as both an independent scientific discipline and an evidence-based practice profession. ${ }^{1}$ Nursing research competence (NRC), which refers to individual

\section{Strengths and limitations of this study}

The scoping review proposed in this protocol will achieve a high level of rigour, as it will adhere to Arksey and 0'Malley's methodological framework, Levac et a/s recommendations and the Preferred Reporting Items for Systematic Reviews and MetaAnalyses extension for Scoping Reviews checklist.

- The scoping review will include different English and Chinese databases.

- The COnsensus-based Standards for the selection of health Measurement INstruments methodology will be used to simultaneously evaluate the methodological quality of studies on measurement properties of the instruments and the quality of all instruments identified.

- This scoping review may fail to include relevant literature, which has been published outside of the searched databases.

- This scoping review is limited to include studies published in English or Chinese.

ability to conduct nursing research activities, ${ }^{2}$ is crucial for generating the high-quality nursing research required for the development of the nursing discipline/profession. ${ }^{3}$ NRC is a particularly necessary competency for nursing researchers and academics to have, as they are most responsible for developing high-quality nursing research. However, clinical nurses are also increasingly expected to be equipped with NRC, so that they can contribute to the ongoing development of evidence-based nursing practice, as well as to bring that evidence into practice to help reduce the known gaps between nursing research and practice. ${ }^{4}$ Furthermore, strengthening NRC among student nurses is important because they will work as future clinical nurses and nursing academics. ${ }^{5}$ For all of these reasons, NRC, which is vital for the development of the nursing discipline and evidence-based nursing practice, has been getting increased attention within many areas of nursing. 
The measurement of NRC is important for research, education and management related to NRC. In research, NRC instruments are required to assess the state of NRC for a given individual nurse or for a nursing organisation; and to explore factors which influence NRC. ${ }^{67}$ In education, NRC instruments can be used to evaluate courses or training programmes for nursing research. ${ }^{5}$ The evaluation of NRC of clinical nurses using validated instruments can also provide nurse managers with evidence for nursing management related to nursing research, such as for determining which nurses within the organisation are most capable of assisting with the organisation's research projects. ${ }^{6}$ Therefore, instruments for measuring NRC are important for related research, education and management.

Some related instruments have emerged during the past decade, based on varying definitions of NRC and diverse contexts for the instruments' uses. ${ }^{356}$ Corchön defined NRC as nursing 'research skills and knowledge, interest and attitudes', and they developed corresponding instruments measuring NRC. ${ }^{3}$ Some definitions of NRC and corresponding instruments focus more on research knowledge and skills. For example, Pan et al developed an instrument measuring 'Research Competence for Clinical Nurses' based on the definition of NRC (ie, the ability to identify problems and formulate research questions, search and critically review literature, design and implement research, analysis data, and write research reports). ${ }^{8}$ Qiu et al defined NRC as the 'ability (1) to systematically summarise clinical experiences as well as literature, in order to find researchable problems and formulate innovative research questions of relevance to nursing, (2) to collect, analyse and explain data related to those questions and (3) to apply knowledge accumulated in this way to solve problems innovatively'; they developed an instrument of Research Competency for Nursing Students. ${ }^{5}$ Some researchers simply define NRC as evidence-based practice competence; the corresponding instruments developed by these researchers tend to focus on domains relevant to evidence-based practice. ${ }^{9}$ These different definitions indicate that there is still no consistent definition of and instrument for measuring NRC.

In this study, we will define NRC as 'individual ability to conduct nursing research activities'. ${ }^{2}$ Since a systematic review has already been conducted on instruments measuring knowledge, skills and attitudes for evidencebased practice among nurses, ${ }^{10}$ the literature on instruments designed solely for measuring evidence-based practice competence will be excluded in this study; however, literature on instruments measuring evidencebased practice and research competence will still be included. There has been also a systematic review of instruments measuring nurses' attitudes towards research utilisation. ${ }^{11}$ However, to our knowledge, no comprehensive knowledge synthesis for instruments of NRC has ever been conducted.

In order to assess NRC in specific contexts, the selection of an appropriate, well-validated and reliable instrument is vital for the credible assessment of NRC in that context. ${ }^{12}$ Although an increasing number of instruments of NRC have emerged, an overview of all available NRC instruments; as well as an evaluation of these various instruments for guiding instrument selection and further instrument development; is still lacking. Therefore, the purpose of this protocol is to design a comprehensive and rigorous scoping review of the instruments for measuring NRC. The scoping review will offer implications for researchers on the NRC instrument development and improvement and/or provide guidance for nurse educators, managers and researchers on the selection of the appropriate NRC instrument to measure NRC in a specific context.

This scoping review will be able to answer the following questions: (1) What instruments for measuring NRC have been developed and how were they used in related studies? (2) Are there any well-validated and reliable instruments for measuring NRC? (3) If there is more than one well-validated and reliable instrument for measuring NRC, are there circumstances under which certain instruments are more appropriate for measuring NRC than the other instruments? (4) What are the differences between NRC instruments designed for different groups (eg, clinical nurses, nursing students)? and (5) What are potential directions for the future development and improvement of NRC instruments?

\section{METHODS}

\section{Objectives}

The two objectives of the proposed scoping review are:

1. To identify, evaluate, compare and summarise the instruments developed to measure NRC.

2. To provide an overview of the use of all the instruments that have been developed for measuring NRC.

\section{Study design}

Considering that the aim of the proposed research is to provide an overview of the evidence; and to identify and analyse the knowledge gaps within the topic of NRC instruments; a scoping review methodology is appropriate for this aim. ${ }^{13}$ Therefore, the study design will be a scoping review following: (1) Arksey and O'Malley's methodological framework, ${ }^{14}$ (2) Levac et als recommendations for using this framework ${ }^{15}$ and (3) the Preferred Reporting Items for Systematic Reviews and Meta-Analyses extension for Scoping Reviews checklist. ${ }^{16}$

\section{Search strategy}

A systematic search will be performed between 1 December 2020 and 31 December 2020, in eight English databases (ie, Cochrane library, CINAHL, EMBASE, PubMed, PsycINFO, Scopus, ERIC, ProQuest Dissertations \& Theses Global) and two Chinese databases (ie, CNKI and WANFANG DATA). The inclusion of these databases will help ensure the conduction of a comprehensive search which will include as much relevant literature as possible. The inclusion of Chinese databases is 
also an effort for a more comprehensive literature search, given that the research team includes four researchers whose first language is Chinese. A string of keyword search terms relating to 'nursing', 'research', 'competence' and 'instrument' will be used to identify relevant literature. An example of the search strategy in PubMed is shown in online supplemental table S1. The search strategy for Chinese databases is shown in online supplemental table S2.

\section{Inclusion criteria}

Papers will be included if they: (1) were published during 1999-2020 (We limit the time frame to 19992020 is because the most related studies in this area were published after 1999 and we will conduct the literature search between 1 December and 31 December 2020) ; (2) are either dissertations or original research studies published in peer-reviewed journals; (3) include at least one instrument for measuring NRC (ie, literature describing the development of an NRC instrument; literature describing the psychometric testing of an NRC instrument; and/or literature in which an instrument was used to measure the NRC) and (4) have full-text availability.

\section{Study screening}

References identified by the search strategy will be copied into EndNote. Duplicates will be detected and removed, first using EndNote's deduplicating capabilities, followed by manual screening. ${ }^{17}$ Study screening will be conducted by two reviewers independently, using Covidence software. Reviewers will first use the titles and abstracts to screen studies. After the title and abstract screening, the full texts will be checked against the inclusion criteria by two reviewers, independently. In case of disagreements between two reviewers, a third reviewer will make the decision regarding the inclusion of the article. Reference lists of included papers will be checked to identify potentially relevant articles. Those added articles will be screened by using the screening process described above.

\section{Data extraction}

Two reviewers will independently conduct the data extraction, using predesigned data extraction tables (online supplemental tables S3-S6). For different studies, different information will be extracted (see figure 1). A detailed description of all included instruments will be extracted and shown in online supplemental table

Literature meeting the inclusion criteria

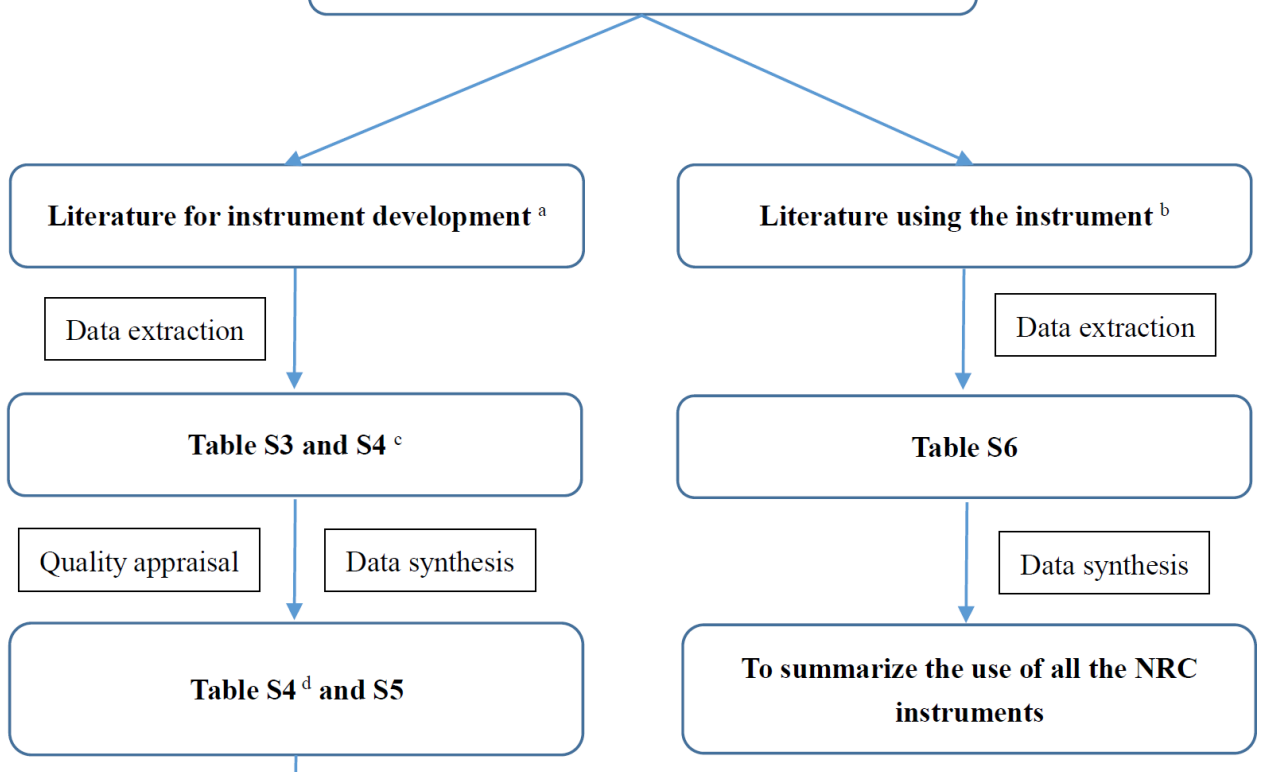

To identify, evaluate, compare, and summarize the NRC instruments

Figure 1 The process of data extraction and synthesis. The included literature will be categorized into two types: ${ }^{a}$ 'Literature for instrument development' refers to the articles focusing on the development and psychometric properties testing of the NRC instrument; 'biterature using the instrument' refers to the articles using the NRC instrument to measure NRC as a study variable. ${ }^{C}$ The data related to measurement properties of the included instrument results will be shown in online supplemental table S4 (Result columns). 'Evaluation results of methodological quality of instrument development and psychometric testing studies will be shown in online supplemental table S4 (Methodological quality columns). NRC, nursing research competence. 
S3, including: name of the instrument, developer (ie, author(s), year developed), construct(s), country of development (language), targeted population, mode of administration, (sub)scale(s)/content(s) (number of items), response options and range of scores/scoring. Psychometric properties of the included instrument will be extracted and presented in online supplemental table S4 (Result columns). ${ }^{18}$ Characteristics of the included studies using the instrument of NRC will be extracted in online supplemental table S6, including: author, year, location, study aim, design/intervention, participants, sample size, the instrument of NRC and results related to NRC.

\section{Quality appraisal}

Considering the aims of this scoping review, the evaluation of the instruments is more important than the quality appraisal of the included studies. Therefore, COnsensusbased Standards for the selection of health Measurement INstruments (COSMIN) methodology will be used to evaluate the methodological quality of the included studies on measurement properties of the instruments, as well as on the quality of all the instruments identified. ${ }^{12}$ The COSMIN methodology is primarily designed for improving the instrument selection of patient-reported outcomes measures, but it can also be adopted and used on other types of outcome measurement instruments. ${ }^{19}$ While using the COSMIN guidelines in our scoping review, some adaptions will be made based on the detailed descriptions of COSMIN guidelines and the characteristics of the construct of interest, that is, NRC. ${ }^{12}$ For example, the 'patients' will be replaced with 'nursing students or nurses' in the COSMIN risk of bias checklist. All adaptions will be discussed in our research group and reviewed by two professors experienced in nursing research.

As the methodological quality of studies on the measurement properties of an instrument is important for the quality of the instrument, COSMIN standards ${ }^{2021}$ will be used to evaluate studies on the methodological quality of an instrument's development and studies of psychometric testing included in this scoping review. The corresponding evaluation results will be shown in online supplemental table S4 (Methodological quality columns). The COSMIN criteria ${ }^{18}$ will be used to evaluate the results of the psychometric properties (Results columns in online supplemental table S4), in order to show the quality of the instruments identified (Rating columns in online supplemental table S4). The synthesised evidence on the quality of each instrument will be graded by using the modified Grading of Recommendations Assessment, Development and Evaluation (GRADE) approach in the COSMIN manual and displayed in online supplemental table S5. ${ }^{12}$

Included studies which only use an instrument to measure NRC (as a study variable), but which do not include contents of instrument development and/or measurement properties testing, will not be appraised.
However, these studies will still be included and analysed in online supplemental table $\mathrm{S} 6$, in order to construct an overview of the usage of all NRC instruments (figure 1).

\section{Data synthesis}

The number of the instruments identified and the numbers of the studies using each specific NRC instrument will be calculated; this analysis will provide an overview of the various NRC instruments. The data extracted for the description and evaluation of all instruments will be reported in data extraction tables (online supplemental tables S3 and S4) to facilitate the synthesis of the evidence, and to provide a visual comparison of all identified NRC instruments. The evidence will be summarised based on the contents of online supplemental table S4. The quality of evidence will be graded by using the modified GRADE approach ${ }^{12}$; these results will be shown in online supplemental table S5, which will indicate the evaluation results of each measurement property of every instrument.

\section{Patient and public involvement}

No patient or public involvement.

\section{Ethics and dissemination}

Ethical approval is not required for this protocol, nor for the scoping review. The scoping review will provide valuable information on the measurement of NRC which is important for research, education and management related to NRC. We will share the findings of this scoping review at a national or international conference and in a peer-reviewed journal article.

\section{DISCUSSION}

To our knowledge, this will be the first scoping review of instruments measuring NRC, as well as the first scoping review to report the characteristics and psychometric qualities of these instruments. Our scoping review will identify, describe, evaluate and compare all instruments for measuring NRC. The findings based on the COSMIN methodology will be used to determine if there is (are) a well-validated and reliable instrument(s) for measuring NRC of a targeted population. This scoping review will also provide recommendations for the selection of the most appropriate instrument (given specific research circumstances), and it will discuss implications for future studies on the development and improvement of instruments for measuring NRC.

\section{Twitter Aimee R Castro @AimeeRCastro}

Contributors Author contributions Study design: QC and ST. Study supervision: $\mathrm{ST}$ and $\mathrm{CH}$. Manuscript writing: QC, ARC and $\mathrm{CH}$. Critical revisions for important intellectual content: ST, $\mathrm{CH}$ and ARC.

Funding The authors have not declared a specific grant for this research from any funding agency in the public, commercial or not-for-profit sectors.

Competing interests None declared.

Patient consent for publication Not required.

Provenance and peer review Not commissioned; externally peer reviewed. 
Supplemental material This content has been supplied by the author(s). It has not been vetted by BMJ Publishing Group Limited (BMJ) and may not have been peer-reviewed. Any opinions or recommendations discussed are solely those of the author(s) and are not endorsed by BMJ. BMJ disclaims all liability and responsibility arising from any reliance placed on the content. Where the content includes any translated material, BMJ does not warrant the accuracy and reliability of the translations (including but not limited to local regulations, clinical guidelines, terminology, drug names and drug dosages), and is not responsible for any error and/or omissions arising from translation and adaptation or otherwise.

Open access This is an open access article distributed in accordance with the Creative Commons Attribution Non Commercial (CC BY-NC 4.0) license, which permits others to distribute, remix, adapt, build upon this work non-commercially, and license their derivative works on different terms, provided the original work is properly cited, appropriate credit is given, any changes made indicated, and the use is non-commercial. See: http://creativecommons.org/licenses/by-nc/4.0/.

\section{ORCID iDs}

Qirong Chen http://orcid.org/0000-0003-1908-290X

Chongmei Huang http://orcid.org/0000-0001-6794-0724

Aimee R Castro http://orcid.org/0000-0002-6461-0866

Siyuan Tang http://orcid.org/0000-0001-9940-5072

\section{REFERENCES}

1 Polit DF, Beck CT. Nursing research: generating and assessing evidence for nursing practice. 9 edn. Philadelphia: Lippincott Williams \& Wilkins, 2012.

2 Chen $\mathrm{Q}$, Sun $\mathrm{M}$, Tang $\mathrm{S}$, et al. Research capacity in nursing: a concept analysis based on a scoping review. BMJ Open 2019;9:e032356.

3 Corchön S. The development, implementation and evaluation of a strategy to enhance nursing research in clinical nursing. University of Sheffield, 2009.

4 Pearson A, Field J, Jordan Z. Evidence-based clinical practice in nursing and health care: assimilating research experience and expertise. John Wiley \& Sons, 2009.

5 Qiu C, Feng X, Reinhardt JD, et al. Development and psychometric testing of the research competency scale for nursing students: an instrument design study. Nurse Educ Today 2019;79:198-203.

6 Pan YH, Cheng JL. The development of research competence scale for clinical nurses. Chin Nurs Res 2011;25:1205-8.
7 Corchon S, Portillo MC, Watson R, et al. Nursing research capacity building in a Spanish Hospital: an intervention study. J Clin Nurs 2011;20:2479-89.

8 Pan Y. 护理人员科能力自评量表的修订 [The development of nursing research competence of nurses self-evaluation scale]. Shanxi Medical University, 2011.

9 Mallidou AA, Borycki E, Frisch N, et al. Research competencies assessment instrument for nurses: preliminary psychometric properties. J Nurs Meas 2018;26:E159-82.

10 Leung K, Trevena L, Waters D. Systematic review of instruments for measuring nurses' knowledge, skills and attitudes for evidencebased practice. J Adv Nurs 2014;70:2181-95.

11 Frasure J. Analysis of instruments measuring nurses' attitudes towards research utilization: a systematic review. J Adv Nurs 2008;61:5-18.

12 Mokkink LB, Prinsen CAC, Patrick DL. COSMIN methodology for systematic reviews of Patient-Reported Outcome Measures (PROMs) - user manual, 2018a. Available: www.cosmin.nl

13 Munn Z, Peters MDJ, Stern C, et al. Systematic review or scoping review? guidance for authors when choosing between a systematic or scoping review approach. BMC Med Res Methodol 2018;18:143.

14 Arksey H, O'Malley L. Scoping studies: towards a methodological framework. Int J Soc Res Methodol 2005;8:19-32.

15 Levac D, Colquhoun H, O'Brien KK. Scoping studies: advancing the methodology. Implement Sci 2010;5:69.

16 Tricco AC, Lillie E, Zarin W, et al. PRISMA extension for scoping reviews (PRISMA-ScR): checklist and explanation. Ann Intern Med 2018;169:467-73.

17 Bramer WM, Giustini D, de Jonge GB, et al. De-duplication of database search results for systematic reviews in endnote. $J$ Med Libr Assoc 2016;104:240-3.

18 Prinsen CAC, Mokkink LB, Bouter LM, et al. COSMIN guideline for systematic reviews of patient-reported outcome measures. Qual Life Res 2018;27:1147-57.

19 Mokkink LB, Prinsen CAC, Bouter LM, et al. The consensus-based standards for the selection of health measurement instruments (COSMIN) and how to select an outcome measurement instrument. Braz J Phys Ther 2016;20:105-13.

20 Mokkink LB, de Vet HCW, Prinsen CAC, et al. COSMIN risk of bias checklist for systematic reviews of patient-reported outcome measures. Qual Life Res 2018b;27:1171-9.

21 Terwee CB, Mokkink LB, Knol DL, et al. Rating the methodological quality in systematic reviews of studies on measurement properties: a scoring system for the COSMIN checklist. Qual Life Res 2012;21:651-7. 